\title{
An Automated Vehicle License Plate Identification System Based on LabVIEW
}

\author{
Fuhai Huang \\ School of information science and engineering, LinYi University, LinYi 276000, China \\ hfh8130@163.com
}

Keywords: License plate detection, Digits segmentation, OCR.

Abstract. In this paper, an automated vehicle license plate identification system is implemented based on LabVIEW. The process includes three parts: vehicle license plate localization, characters segmentation and characters recognition. We used color feature to detect license plate location, and then segmentation of characters used projection, and characters recognition used OCR at last. According to the results, the recognition efficiency and accuracy of the system are favorable.

\section{Introduction}

In recent years, with the increasing number of vehicle, license plate recognition (LPR) is more and more widely used. For example, parking management, traffic safety, vehicle tracking and other fields [1].

Realization of the license plate recognition includes three stages: localization and extraction of license plate, character segmentation and character recognition. License locating is the difficulty of the license plate recognition. There are many types of license plates, which vary in color, size and shape. In addition, weather conditions, lighting, license plate fouling and other factors also affect recognition results [2]. The role of license plate location is also very important, the accuracy of location will directly affect the accuracy of subsequent processing results. There are many ways to locate the license plate include method based on texture analysis [3], edge extraction [4], morphological filtering [5], color feature [6] and so on. The method of character segmentation is projection. At present, some new methods have appeared, such as HMM [7], which extract characters, train and classify. Character recognition can be done using model matching and OCR methods. The flowchart of VLP recognition system as shown in Fig. 1.

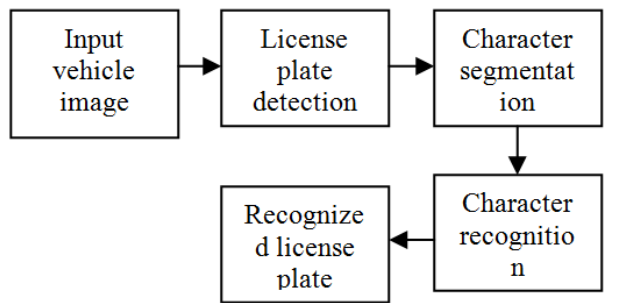

Fig. 1 The flowchart of VLP recognition system

In this paper, license plate localization is based on color feature. This method has better results under the condition of better illumination. In order to let the light stability can increase lighting equipment. The projection method is used in character segmentation, and the advantage of this method is insensitivity to light [8]. Character recognition uses OCR. It's easy to recognize character training and achieve better results in LabVIEW.

\section{Localization and Extraction of License Plate}

As examples, the plate has white words on blue background located in this paper. The basic method of localization is: 1) First of all, according to the statistical characteristics of plates with the blue pixels in the input image, find the most points for pixels respectively to the blue point and search down to meet the conditions, it will be a number of license plate candidate regions in the horizontal direction, and the starting line and end line of each candidate region. 2) Then, from the candidate area, 
columns are searched for eligible blue pixels to get their start and end columns, further reducing the number of candidate constituencies. 3) Finally, count the number of white spots in the remaining candidate area. After multiple positioning, the ratio of length to width and the ratio of blue to white pixels of each candidate area can be obtained, and the eligible candidate area is taken as the final license plate area. Result of the license plate that is segmented by this method is shown in Fig. 2.

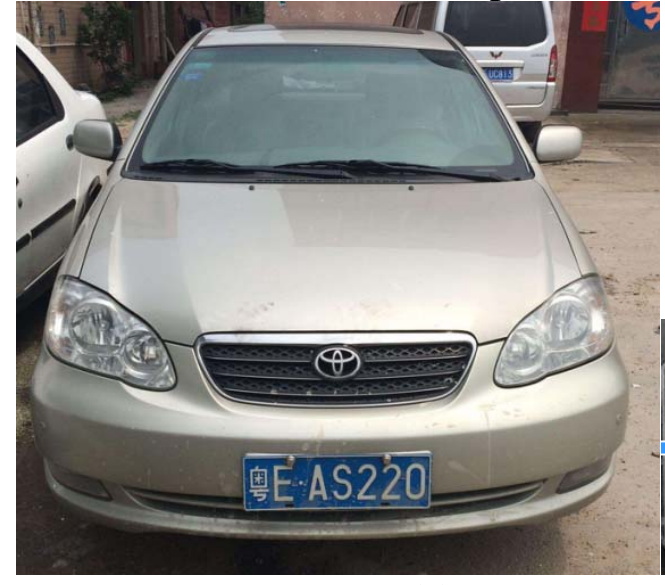

(a)

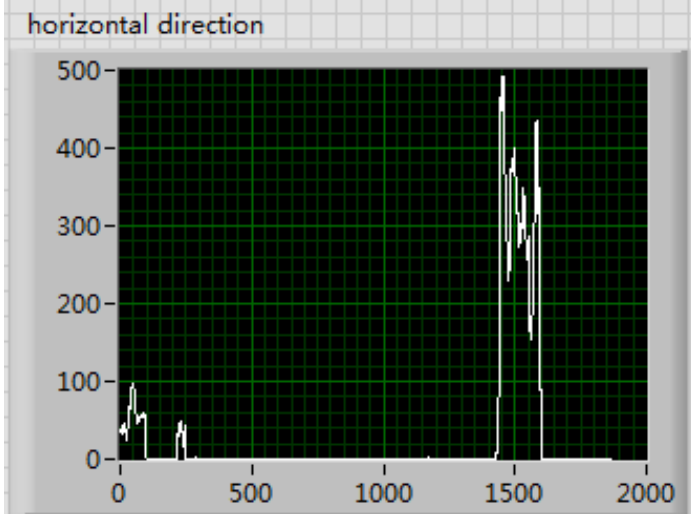

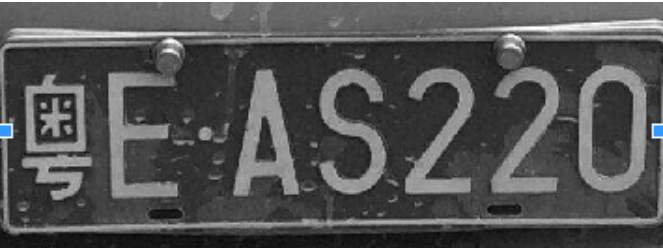

(b) vertical direction

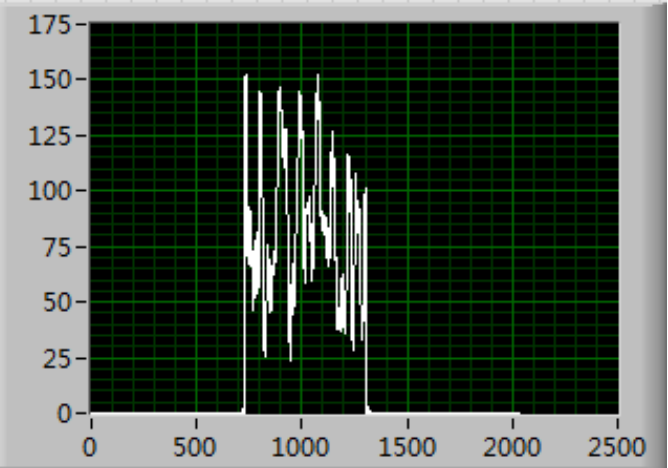

(c)

Fig. 2 Image capture and plate localization:(a) input vehicle image and (b) plate localization, (c) blue pixel statistics along horizontal and vertical directions

\section{Character Segmentation}

Before the characters are segmented, we convert the license plate region to a binary image. IMAQ Auto BThreshold, an automatic threshold function, is provided in LabVIEW. This function provides a variety of ways to compute thresholds, as such clustering, metric, inter-class variance. There are some differences in the handling of various methods. In this paper, the metric method is adopted. Using morphological methods and statistics the area of the Unicom domain can remove irrelevant points in the license plate area. Then, the image is inverted in order to reduce the amount of computation and final character recognition. Statistics the number of black points in the vertical and horizontal directions. The character position can be obtained based on statistical results. These processing results are shown in Fig. 3.

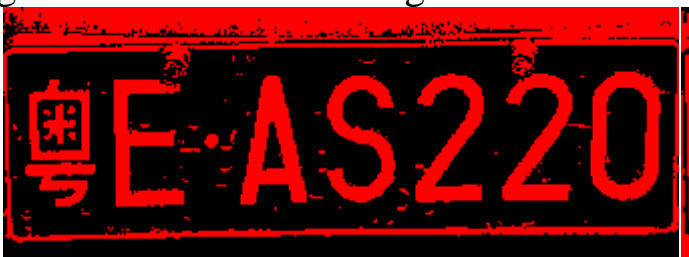

(a)

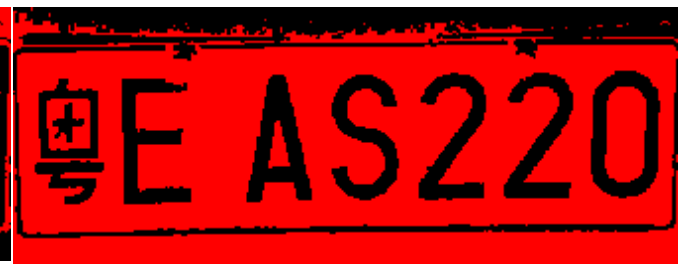

(b) 


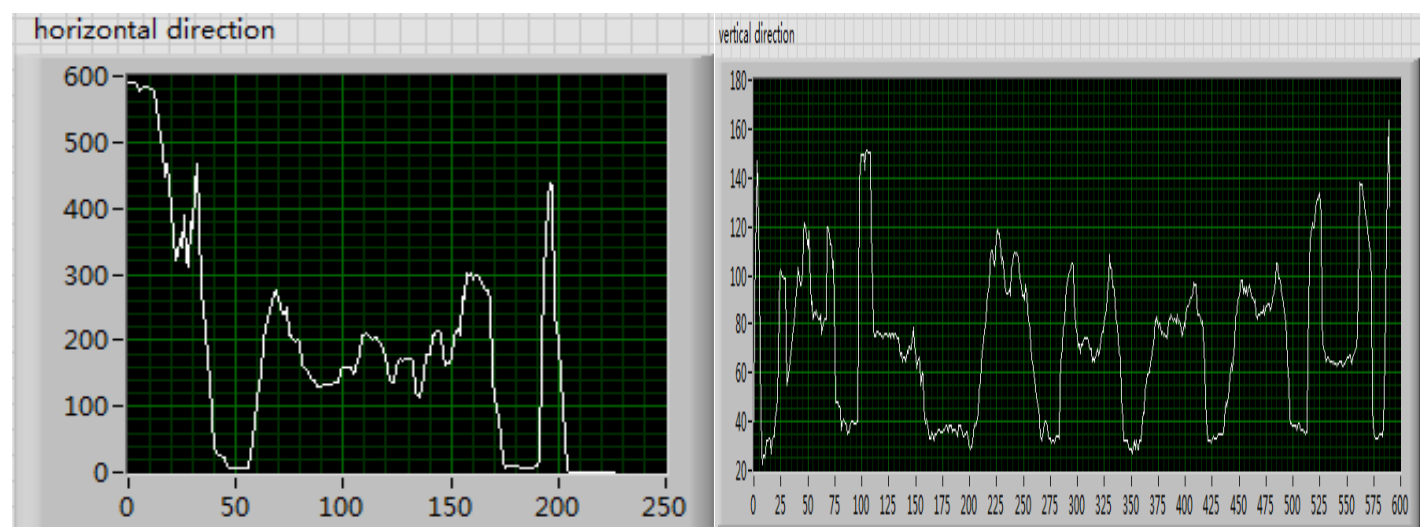

(c)

(d)

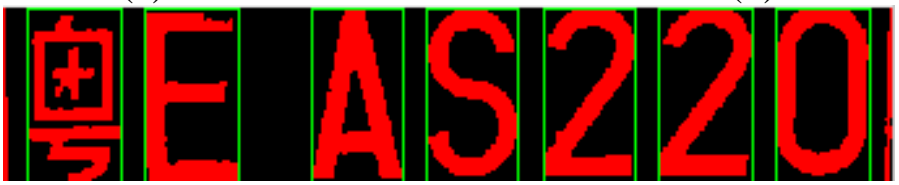

(e)

Fig. 3 character segmentation and extraction: (a) binary image, (b) morphological processing and negation, (c) and (d) black pixel statistics along horizontal and vertical directions, (e) result of character segmentation

\section{Character Recognition}

Using OCR(Optical Character Recognition) to identify the segmented characters. LabVIEW provides OCR Training tools, we can easily establish a standard sample library. Select samples and train through this tool. The appropriate number of samples and the setting of Acceptance level in the training sample will have an impact on the recognition results.

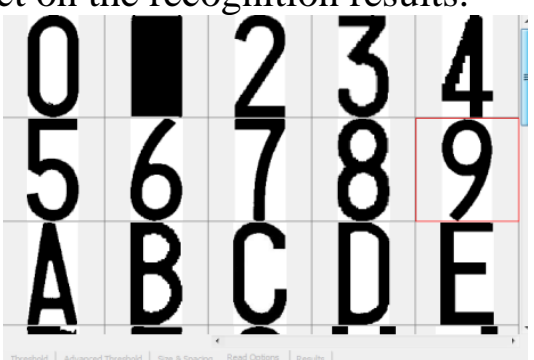

Fig.4 NI OCR Training

In order to further improve the recognition accuracy, the segmented characters are normalized and then sent to the OCR system for recognition. The results are shown in Fig.5.
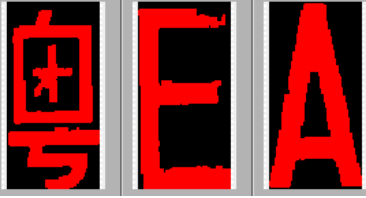

(a)

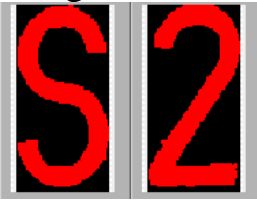

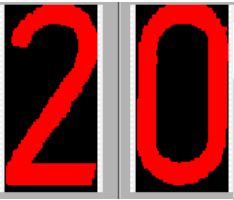

String (OCR/OCV 1)

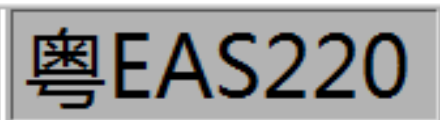

(b)

Fig.5 (a) result of character extraction and normalization, (b) result of character recognition

\section{Conclusion}

By analyzing the processing results of 200 input images, the recognition rate of the system is about 95\%. There are 2 reasons of errors: 1 ) when the color of the car body is close to the color of the license plate, the location of the license plate is easy to fail; 2) The quality requirements of input images are higher, the license plate unable to located because low resolution. The implementation of 
the system based on NI LabVIEW, its rich image processing function and graphical language also reduce the development cycle and improve the development efficiency.

\section{References}

[1]. L. Miao, F. Wang, and H. Wang, “Automatic License Plate Detection Based on Edge Density and Color Model”, Proc. IEEE Control and Decision Conf. (CDC 09), 2009, pp. 3718-3721.

[2]. H Rajput, T Som, and S Kar, "Automated Vehicle License Plate Recognition System", Computer, 2015, pp. 56-61.

[3]. Vladimir Shapiro, Georgi Gluhchev, and Dimo Dimov, "Towards a Multinational Car License Plate Recognition System”, Machine Vision and Applications, 2006, 17:173-183.

[4]. Danian Zheng, Yannan Zhao, and Jiaxin Wang, “An efficient method of license plate location”, Pattern Recogn. Lett. 2005:2431-8.

[5]. Zhu Wei-gang, Hou Guo-jiang, and Jia Xing, “A study of locating vehicle license plate based on color feature and mathematical morphology”, Signal Processing, 2002 6th International Conference on, Volume: 1

[6]. Vahid Abolghasemi, Alireza Ahamadyfard, “An edge-based color-aided method for license plate detection”, Image and Vision Computing, 2009, 27(8):1134-1142

[7]. S. ADEBAYO DARAMOLA, E. ADETIBA, “AUTOMATIC VEHICLE IDENTIFICATION SYSTEM USING LICENSE PLATE”, IJEST, 2011, 2: 1712-1719 VOL.3.

[8]. LEARNING-BASED APPROACH FOR LICENSE PLATE RECOGNITION,K.K.KIM, K.I.Kim, Ieee Signal Processing Society Workshop, 2000 , 2 :614-623 VOL.2. 